\title{
Therapeutic HPV vaccines
}

Gemma Hancock $^{a^{*}}$, Karin Hellner ${ }^{b}$, Lucy Dorrell ${ }^{a, b}$

${ }^{a}$ Nuffield Department of Medicine, University of Oxford and Oxford NIHR Biomedical Research Centre, NDM Research Building, Old Road Campus, Headington, Oxford, UK

${ }^{\mathrm{b}}$ Nuffield Department of Obstetrics and Gynaecology, University of Oxford, Women's Centre, John Radcliffe Hospital, Oxford, UK.

Gemma.hancock@ndm.ox.ac.uk

Lucy.dorrell@ndm.ox.ac.uk

Karin.hellner@obs-gyn.ox.ac.uk

* Corresponding author

Gemma.hancock@ndm.ox.ac.uk, 01865612914 
Abstract

High-risk human papillomavirus (hrHPV) infection is known to be a necessary factor for cervical and anogenital malignancies. Cervical cancers account for over a quarter of a million deaths annually. Despite the availability of prophylactic vaccines, HPV infections remain extremely common worldwide. Furthermore, these vaccines are ineffective at clearing pre-existing infections and associated pre-invasive lesions. As cervical dysplasia can regress spontaneously, a therapeutic HPV vaccine that boosts host immunity could have a significant impact on the morbidity and mortality associated with HPV. Therapeutic vaccines differ from prophylactic vaccines in that they are aimed at generating cell-mediated immunity rather than neutralising antibodies. This review will cover various therapeutic vaccine strategies in development for the treatment of HPV-associated lesions and cancers.

Keywords: High-risk human papillomavirus, HPV-associated malignancies, therapeutic vaccine, viral vector, DNA vaccine, bacterial vector 


\section{(A) Why is there a need for a therapeutic vaccine}

HPV was discovered to be the causative agent of cervical cancer in the 1970 s by the Zur Hausen group [1]. The majority of HPV infections are transient and subclinical due to rapid immune clearance. However persistent highrisk HPV (hrHPV) infection may lead to the development of precancerous lesions. HPV-16 and -18 have been identified as the two most prevalent hrHPV types and are accountable for approximately 62.6 and $15.7 \%$, respectively, of invasive cervical cancers [2]. Additionally, these two hrHPV types are responsible for $80-86 \%$ of vulvar and vaginal cancers, $89-95 \%$ of oropharyngeal cancers, $93 \%$ of anal cancers, and $63-80 \%$ of penile cancers [3].

In the trials that led to the approval of the prophylactic vaccines, Gardasil and Cervarix, these vaccines were found to provide nearly $100 \%$ protection against persistent cervical infections with HPV types 16 and 18 [4]. The vaccines are also highly efficacious in preventing precancerous cervical lesions (cervical intraepithelial neoplasia, $\mathrm{CIN}$ ) caused by these genotypes [5,6]. Despite this, there is still a considerable population suffering from hrHPV infections and associated disease worldwide. There are several reasons for this;

1. Limited global vaccine uptake. By the end of 2016 , nearly 70 countries had introduced a prophylactic HPV vaccine into their national immunisation schedule [7]. However, the countries with the highest morbidity owing to cervical cancer and anogenital warts were also the least likely to have introduced prophylactic vaccination. By mid-2016, only $8 \%$ of low to-middle income countries had introduced the HPV vaccine, compared to $71 \%$ of high-income countries [8].

2. Limited cross-protection. The current bivalent and quadrivalent vaccines provide $100 \%$ protection against infection with HPV-16 and 18 [4]. However non-vaccine hrHPV types are responsible for about $30 \%$ of cervical cancers [9]. Two independent analyses of the Phase III studies FUTURE I and FUTURE II assessed cross-protection afforded by the quadrivalent vaccine. Brown [10] and Wheeler [11] reported a reduction in the incidence of HPV31/45 infection by 40.3 and $31.6 \%$ respectively and of 31/33/45/52/58 infection by 25.0 and $17.7 \%$ respectively. They also reported similar reductions in the incidence of HPV31/45 CIN1-3 by 43.6\% and $22.2 \%$ and HPV31/33/45/52/58 CIN1-3 by $29.2 \%$ and $18.8 \%$ respectively in the naïve population. 
3. Prophylactic vaccines provide no therapeutic benefit and are only effective in HPV-naïve individuals as their mechanism of action is the induction of antibodies against the L1 capsid protein. L1 is only expressed in the granular epithelium just prior to viral shedding and therefore current prophylactic vaccines are unable to eliminate pre-existing infections and associated lesions. Clearance of infected cells is dependent on induction of a cell-mediated immune response $[12,13]$.

4. Cost and requirement for a cold chain. This restricts wide scale deployment in developing countries, where $\sim 87 \%$ of cervical cancer deaths occur [14].

5. Because of the long time required for the development of pre-cancerous lesions, it is estimated that an impact on cancer incidence may not become apparent for at least 20 years from the implementation of mass HPV vaccination.

Women with low-grade disease (CIN1), which is known to have high regression rates [15,16], are often observed and cervical cytology repeated annually until disease progression is detected $[17,18]$. For high-grade disease (CIN2-3) treatment options include excisional approaches using electric loops (loop electrosurgical excision procedure (LEEP) or large loop excision of the transformation zone (LLETZ)), carbon dioxide lasers or scalpels (cold knife) to perform conisation, in which the entire transformation zone (TZ) is removed. Although there is no difference in haemorrhage or CIN recurrence between the three conisation approaches [18], adverse obstetric outcome, such as preterm birth and second trimester miscarriage, is increased with cold knife conisation [1921]. Local destruction of the TZ by cryotherapy or laser ablation is more time-consuming than excisional procedures and as these techniques do not yield tissue for histological assessment, they are only appropriate after diagnosis via biopsy and when no invasion is suspected. Hysterectomy is not part of standard therapy for $\mathrm{CIN}$ and only considered in certain circumstances. Treatment of high-grade cervical lesions in LMIC countries has been estimated to cost $\$ 12.6$ million annually [22]. The infrastructure required to deliver and monitor the efficacy of ablative treatment, which is a major challenge for LMICs carrying the vast burden of HPV associated disease, makes non-surgical therapies for pre-invasive hrHPV lesions highly desirable.

Thus, there is an urgent need to develop therapeutic vaccines against hrHPV which could have an immediate impact on women who are already infected by preventing the progression of low-grade disease, inducing regression of existing lesions, and/or preventing recurrence following treatment. hrHPV infection is an attractive 
target for immunotherapy because the pre-invasive stages are well-defined and progression to cancer typically takes 10-30 years, providing ample opportunity for therapeutic intervention. Furthermore, spontaneous regression of high grade (cervical intraepithelial neoplasia, CIN2/3) lesions occurs in $30 \%$ of cases [23] indicating that host immune responses can reverse the course of disease.

\section{(A) The requirement for a cell mediated immune response}

While humoral immune responses have important preventive utility, several lines of evidence suggest that cellmediated immune responses play a crucial role in regression of precancerous lesions and clearance of infection. Firstly, persistent hrHPV infections and pre-invasive lesions are significantly more frequent in immunosuppressed states such as untreated HIV infection [24] or receipt of anti-rejection therapy posttransplant [25]

Spontaneous regression of high-grade CIN has been reported to correlate with cell-mediated immune responses. Regressing lesions are typically infiltrated by $\mathrm{CD}^{+}{ }^{+} \mathrm{T}$ cells specific for the oncoproteins, E6 and E7. In addition, $\mathrm{CD}^{+} \mathrm{T}$ cell helper responses to $\mathrm{E} 2$, a key regulator of transcription, have been detected in individuals following regression of CIN [26-30]. Increased CD4:CD8 ratios in the stroma have also been described. By contrast, reduced numbers of $\mathrm{CD}^{+} \mathrm{T}$ cells are present in persistent and/or progressing CIN lesions [31].

Regulatory T cells (Tregs) have been found in persistent HPV infections. The frequency of Tregs increases with the size of genital warts [32]. Clinical studies have suggested that Tregs may facilitate both viral persistence $[33,34]$ and disease progression [35].

Patients with AIN (anal intraepithelial neoplasia) and VIN (vulval intraepithelial neoplasia) treated with Imiquimod, a Toll-like receptor (TLR)-7 agonist that induces innate immune responses, developed HPV16-specific $\mathrm{CD} 4^{+} \mathrm{T}$ cell responses that correlated with remission of lesions [36].

Cross-sectional human studies provide only a snapshot of what is a dynamic process however, animal studies have been informative in revealing the immunological events of the entire cycle from infection to regression. The canine oral papillomavirus model confirms observations in human studies that the predominant infiltrate 
associated with regression of lesions comprises $\mathrm{CD} 4^{+} \mathrm{T}$ and $\mathrm{CD} 8^{+} \mathrm{T}$ cells, specific for the early proteins E2 and E6 [37].

In summary, induction of potent cellular immune responses to E6 and E7, and possibly other viral antigens, is likely to be crucial to the efficacy of a therapeutic HPV vaccine.

\section{(A) How to induce a cell mediated immune response by vaccination}

The generation of virus-specific cytotoxic T cells (CTLs), which target and kill infected cells, and T helper cells, which secrete cytokines and orchestrate the maturation of CTLS and B cells is dependent on priming by professional antigen-presenting cells (APCs) such as dendritic cells (DCs). Thus, therapeutic vaccines require rational design to achieve concentrated antigen delivery to DCs and DC activation. When a pathogen, or component thereof, penetrates epithelial and mucosal barriers, the first line of defense is the innate immune response. Pathogen-associated molecular patterns (PAMPs) in viral capsids or bacterial membranes are recognized by pathogen recognition receptors (PRRs), such as TLRs on the cell surface. Activation of PRRs leads to the production of proinflammatory cytokines and chemokines and an influx of nonspecific inflammatory cells such as macrophages, DCs, natural killer (NK) cells to the site of infection. The main cytokines involved in an antiviral response are the interferons (IFN- $\alpha$, IFN- $\beta$ and IFN- - ) and the tumour necrosis family (TNF- $\alpha$ and TNF$\beta)$; these have direct antiviral effects as well as immunomodulatory effects. Professional APCs (DCs, monocytes, macrophages and B cells) carrying foreign antigen migrate from the site of infection to the draining lymph nodes, where they present this antigen to naïve T cells in the form of peptides complexed to major histocompatibility complex (MHC) class I or II molecules, initiating the primary adaptive immune response. The defining feature of professional APCs is the expression of co-stimulatory molecules (CD80 and CD86) which are essential for activation of naïve T cells. The MHC class I pathway processes proteins from the cytosol. Many viruses are not tropic for dendritic cells and are therefore not present in the cytosol of professional APCs; however, exogenous antigens can access MHC class I molecules via a process called cross-presentation. Viral antigens derived from phagocytosed/endocytosed apoptotic or necrotic virus-infected cells can leave the vacuole in which they have been engulfed and gain entry to the cytosol, where they are subsequently degraded by the proteasome, and presented by MHC class I to naive $\mathrm{CD} 8^{+} \mathrm{T}$ cells. These cells subsequently proliferate and differentiate into effector 
cells that can directly eliminate virus-infected cells. Upon clearance of the pathogen, a small fraction of $C D 8^{+} T$ cells persist as effector memory cells that patrol the periphery and rapidly upregulate effector molecules upon encounter with cognate antigen. Other $\mathrm{CD}^{+} \mathrm{T}$ cells differentiate into central memory cells, which reside in lymphatic tissue where they are maintained for years.

The MHC class II pathway generates peptides by degradation of exogenous antigens internalized by phagocytosis/endocytosis for presentation to $\mathrm{CD} 4^{+} \mathrm{T}$ cells. In the presence of cytokines such as interleukin 12 (IL12) and intercellular adhesion molecule 1 (ICAM-1), naïve CD4 ${ }^{+}$T cells differentiate into T helper 1 (Th1) cells. Th1 cells release cytokines that provide signals necessary for both the priming of naïve $C D 8^{+} T$ cells and maintenance of their effector functions. Cytokines including OX-40 ligand, IL-10 and transforming growth factor $\beta$ (TGF- $\beta$ ) induce Th2 cell polarization and these cells promote $B$ cell differentiation, proliferation and antibody production in response to extracellular pathogens.

\section{(A) Target antigen}

The HPV genome encodes seven early proteins ( $E 1,2,4,5,6,7,8)$ and two late proteins ( $L 1$ and $L 2)$. $L 1$ and $L 2$ encode the viral capsid proteins which are necessary for the initial infection of the basal layer of epithelium. Following virion internalisation, the E1 and E2 proteins initiate and maintain viral replication. Because they are essential for HPV replication within host cells and are expressed early in the virus life cycle, they are potential targets for vaccines that aim to treat early stages of disease, such as CIN1. Additionally, responses to E2 are associated with regression of CIN and virus clearance $[27,28]$.

E6 and E7 drive oncogenesis by targeting host cell cycle control. E7 binds to and inactivates the retinoblastoma gene product, pRb, leading to uncontrolled cellular proliferation. The tumour suppressor, p53, would normally induce apoptosis in this situation, however E6 binds to p53 resulting in its ubiquitination and subsequent degradation, thereby inhibiting apoptosis. The concerted actions of E6 and E7 result in malignant transformation of HPV-infected cells and uncontrolled tumour growth. E6 and E7 are constitutively expressed in both premalignant and invasive lesions but are absent on healthy cells, making them ideal targets for immunotherapeutic approaches for HPV-induced malignancies. They have therefore been included in most 
therapeutic vaccine candidates developed to date. Additionally, because E6 and E7 are foreign proteins there is minimal risk of induction of immune tolerance.

E5 cooperates with E6 and E7 oncogenes to promote hyper-proliferation of infected cells and is therefore regarded as being crucial for malignant progression. Despite this, few studies have investigated its potential as a target antigen.

\section{(A) Vector of choice}

\section{(B) Nucleic acid based vaccines}

\section{(C) DNA}

DNA vaccination involves the delivery of plasmid DNA encoding a protein of interest into the host's tissues, with subsequent transfection of cells, leading to expression of the transgene and production of proteins that can access the cellular processing machinery. A DNA vaccine typically consists of a bacterial plasmid containing a strong viral promotor, the gene of interest and a polyadenylation/transcriptional termination sequence. After injection, the DNA may be taken up by myocytes or dendritic cells. The bacterial DNA backbone can induce an innate immune response, through recognition of $\mathrm{CpG}$ motifs that are a ligand for TLR9 on dendritic cells, B cells and NK cells [38]. TLR9 ligation triggers a cascade of pro-inflammatory responses with subsequent cytokine production leading to the recruitment and activation of other immune cells.

DNA vaccines are thought to induce an adaptive cellular immune responses by any or all of the following mechanisms.

1. Transfection of somatic cells and subsequent presentation of encoded antigens to $C D 8^{+} T$ cells via the $M H C$ class I pathway. This is more common when the vaccine is delivered by intramuscular injection which tends to result in transfection of myocytes. However, because myocytes are not professional APCs, they are incapable of activating strong specific immune responses. 
2. Direct transfection of professional APCs leading to $M H C$ class I-associated antigen presentation to naïve $C D 8^{+} T$ cells. This is more common when the DNA vaccine is delivered intradermally as it can access Langerhans cells residing in the skin.

3. Phagocytosis of transfected somatic cells by professional APCs, leading to processing and presentation of exogenous antigens via $\mathrm{MHC}$ class II molecules to $\mathrm{CD} 4^{+} \mathrm{T}$ cells helper cells or cross presentation to $\mathrm{CD} 8^{+} \mathrm{T}$ cells.

The advantages of DNA vaccines are: 1 . Simplicity and low cost of manufacture, 2 . thermostability; 3 . repeating dosing is possible due to the absence of anti-vector immune responses, 4. potential to elicit both cellular and humoral immunity, 5 . excellent safety and tolerability in humans. The primary safety concern for DNA vaccines is the potential to integrate into host cellular DNA, however little evidence of this has been found $[39,40]$.

Unfortunately, clinical studies of DNA vaccines have been disappointing and have failed to reproduce the high levels of immunogenicity observed in small animal models. Cellular uptake of naked DNA is very inefficient in vivo and the bulk of the injected DNA remains extracellular [41]. The immunogenicity of DNA vaccines can be improved by optimization of codon-usage to improve expression in vivo [42] and the addition of a leader sequence to target antigens to the endoplasmic reticulum (ER). The ability to incorporate additional genes, into the vector creates opportunities to modulate cellular routing and the subsequent immune response (e.g. IL2[43]). In addition, electroporation (EP), which involves applying brief electric pulses to the skin can greatly enhance vaccine uptake and subsequent antigen delivery by inducing transient permeabilisation of the cell membrane. EP also induces damage to the application site and therefore acts as a form of adjuvant that leads to inflammation and cytokine release with subsequent recruitment of APCs to the area [44]. Immunogenicity of DNA vaccines can also be improved by using them in a heterologous prime-boost vaccination strategy. In the combination of DNA prime viral vector boost, the lack of viral antigens in the DNA vector helps focus the immune response on the key antigen and the additional innate antiviral responses that develop following the viral vector boost result in high magnitude antigen-specific responses [45]. Such a regimen may generate immune responses that are higher in magnitude and better in quality compared with homologous prime boost and avoid anti-vector immunity [46]. 
In 2015, proof of concept for a therapeutic DNA-vectored HPV vaccine was demonstrated in phase Ilb study by Trimble et al [47]. VGX-3100 consists of two DNA plasmids encoding optimised synthetic consensus E6 and E7 genes of HPV-16 and HPV-18. 167 women with histologically confirmed HPV-16/-18 positive CIN2/3 were randomised 3:1 to receive VGX-3100 or placebo, given intramuscularly at 0,4 and 12 weeks, followed by electroporation. Histopathological regression occurred in $49.5 \%$ of vaccinees compared with $30.6 \%$ of placebo recipients (percentage point difference 19.0, $\mathrm{p}=0.034$ ). In a post-hoc analysis, the magnitude of T cell responses to E6 was associated with clinical outcome [47]. This trial addressed two important issues, firstly it demonstrated that a therapeutic vaccine can induce adaptive immune responses in patients with existing disease. Secondly, administration of a vaccine systemically can elicit adaptive immune responses that have a therapeutic effect distally in a mucosal target lesion. A phase III trial (REVEAL) is currently underway to confirm the efficacy, safety, and tolerability of VGX-3100 followed by EP in women with histologically confirmed HPV-16/-18 positive CIN2/3. It aims to recruit 198 patients with an expected completion date of 2020 (NCT03185013). A phase II study of VGX-3100 followed by EP alone or in combination with Imiquimod is also underway in women with HPV16/18positive high-grade lesions of the vulva (NCT03180684).

\section{(C) RNA}

Once administered and internalized by host cells, mRNA transcripts are translated directly in the cytoplasm. Therefore, in contrast to DNA vaccines, RNA vaccines only have to cross the plasma membrane but not the nuclear membrane, which may improve probability of successful transfection. Translation of RNA occurs with the resulting antigens presented by MHC class I molecules. RNA vaccines can also activate the innate immune system by acting as agonists for TLR7 and TLR8 [48]. RNA vaccines are notoriously unstable and as of yet have not been used in any clinical settings for HPV-associated diseases. Encapsulating mRNA in nanoparticles may protect the mRNA from nuclease degradation resulting in enhanced cell uptake and delivery efficiency [49].

\section{(B) Viral vectors}

Because of their natural propensity to transduce their own genetic information into host cells for replication, viruses are attractive modalities for the development of therapeutic HPV vaccines. Non-essential viral genes are replaced with foreign genes coding for immunogenic proteins from pathogens of interest. Recombinant viral 
vectors can then transduce their usual target cell, with subsequent intracellular expression of the encoded antigen. Antigen production is efficient and leads to presentation of both vector and transgene-derived peptides in the context of MHC class I molecules at the cell surface. In addition, viral nucleic acids contain PAMPs that stimulate the innate immune responses. The main drawback of viral vector vaccines is vector-specific immunity: pre-existing antibodies can block uptake of the vector into the target cell, while de novo responses can be amplified with each subsequent vaccine boost and out-compete responses to the transgene. The most extensively tested viral vectors are adenoviruses (Ad), adeno-associated viruses (AAV), alphaviruses and vaccinia virus (such as Modified Vaccinia Ankara, MVA).

\section{(C) Adenoviruses}

Adenoviruses display extensive tropism for many different cell types, which is one of the reasons adenoviruses were pioneered for gene therapy and subsequently developed as vaccine vectors. The adenovirus genome has five early regions, E1A, E1B, E2, E3 and E4. E1A proteins transactivate other viral genes and are critical for the initiation of virus replication; deletion of this region and replacement with the target gene results in replicationdefective virions that are safe for human use. The majority of studies in animals and in humans utilize E1-deleted adenovirus vectors. Additional deletions in the E2, E3 and E4 coding sequences increase the capacity for transgene insertion. Replication-deficient E1/E3-deleted vectors can accommodate approximately $6.5 \mathrm{~kb}$ of foreign DNA. After entering the nucleus, adenoviruses persist episomally, rather than integrating into the host genome, minimising the risk of insertional oncogenesis. Adenovirus vectors can be administered by various systemic and mucosal routes and they are able to transduce both quiescent and actively dividing cells.

As adenoviruses are ubiquitous in humans, pre-existing immunity is common: up to $60 \%$ of adults living in Europe and the USA have high titres of neutralising antibodies to adenovirus type 5 (Ad5), the most commonly used human serotype for gene therapy and vaccine trials. Prevalence increases to over $90 \%$ in individuals living in sub-Saharan Africa therefore a number of alternatives are being evaluated, including rare human serotypes such as Ad26 and Ad35 and non-human adenoviruses from ovine, porcine, bovine and chimpanzee sources. Adenoviruses from chimpanzees (ChAd) were advanced to the clinic because of their low/no seroprevalence in the human population and comparable immunological potency to human Ad5 (reviewed in [50] 
Khan et al [51] investigated the use of rare human adenoviruses, Ad26 and Ad35, encoding E2, E6 and E7 as a fusion protein for the treatment of HPV16/18-related disease. E6 and E7 genes were reordered to abrogate their oncogenic activity. In preclinical studies, robust T cell immunogenicity and protection against tumour challenge was observed upon immunisation of mice. This fusion protein has also been expressed in MVA for use in a prime boost strategy. A phase I trial is planned for 2017 .

While most therapeutic vaccine candidates to date have targeted HPV16 and HPV18, Ragonnaud et al have explored a novel approach to increase the coverage of circulating oncogenic papillomavirus [52]. They designed a papillomavirus ancestor antigen which corresponded to the root of a phylogenetic tree of all oncogenic human and macaque papillomavirus E1 and E2 sequences (CDSE1E2 antigen), as well as two other sequences that were ancestral to the clades containing HPV16/31/35 and HPV18/45 respectively. A molecular T cell adjuvant, the MHC class II invariant chain (li) was fused to the HPV transgene in order to increase antigen presentation in transduced DCs. Fusion of li to various antigens was shown to increase $C D 4^{+} T$ and $C D 8^{+} T$ cell responses [53]. Replication-deficient ChAd3 and ChAd63 vectors encoding CDSE1E2 were shown to induce potent and longlasting CDSE1E2 specific T cell responses in outbred mice. However, CD8 ${ }^{+}$T cell cross-reactivity against HPV16 E1 and E2 was disappointingly limited. A heterologous (ChAd3/ChAd63) prime boost regimen was subsequently tested in female macaques naturally infected with Macaca fascicularis papillomaviruses (MfPVs). All immunized animals developed IFN- $\gamma$ producing $\mathrm{CD}^{+} \mathrm{T}$ cell responses to CDSE1E2, yet also demonstrated poor crossreactivity against circulating papillomaviruses (HPV16, HPV18 MfPV3) and no reduction in HPV virus load [52,54].

\section{(C) Poxviruses}

Poxviruses have a large, stable genome which can support stable expression of large amounts of transgenic DNA whilst retaining transcriptional and translational capacity [55]. Recombinant genes are expressed for $\sim 7$ days before the infected cell is cleared by the immune system [55]. Their entire life cycle takes place in the cytoplasm of somatic human cells, which minimises the risk of insertional mutagenesis. Furthermore, poxviruses infect cells though a passive membrane fusion, thus conferring a broad tropism for mammalian cells, including monocytes and immature myeloid-derived dendritic cells [56]. The extensive use of replication-competent vaccinia and latterly MVA as smallpox vaccines provided evidence for the safety of poxviruses in humans. MVA was highly 
attenuated by $\sim 570$ serial passages of the Ankara strain in primary chick embryo fibroblasts, which resulted in several gene deletions and loss of host immune evasion proteins. Pre-existing immunity is only present in vaccinated individuals, and is therefore diminishing with time. MVA and other attenuated vaccinia strains such as NYVAC are easy to manufacture at a low cost and, have been safely administered by the intradermal, intranasal, intravaginal, and intrarectal routes. MVA and NYVAC are particularly immunogenic when used in heterologous prime-boost strategies.

An MVA vaccine candidate containing the E2 gene from bovine papillomavirus has been evaluated in several Phase I/II clinical trials in patients with established HPV-induced CIN lesions [57-59]. In a phase I/II trial, 36 women with CIN1-CIN3 lesions received MVA-E2 injected directly into the uterus weekly over a 6-week period. $94 \%$ of patients showed complete elimination of precancerous lesions after treatment [57]. The remaining two patients showed regression from CIN3 to CIN1. Following this, in a phase II clinical trial, 56\% of women with HPV16/-18 positive high-grade lesions had a complete regression following vaccination [58].

Recently, this MVA E2 vaccine has been tested in a phase III trial of 1,176 female subjects with HPV-driven oncogenic and non-oncogenic intraepithelial lesions and 180 males with condylomata only. They were injected with MVA-E2 directly into their uterus, urethra, vulva, or anus. Patients were monitored by colposcopy and cytology. Complete histological regression was observed in $89.3 \%$ of female patients and 100 of male patients, after MVA-E2 treatment. No description of E2-specific T cells was provided in any of the studies and because no control vaccine was used in these studies, the true vaccine efficacy is uncertain [60].

Another MVA vectored vaccine, TG4001, developed by Transgene, comprises recombinant MVA encoding HPV16 E6/E7 and human IL2. Safety and efficacy was evaluated in a phase lla trial in 21 patients with HPV16related $\mathrm{CIN} 2 / 3$ lesions; $48 \%$ showed disease regression. Furthermore, of 10 patients showing regression to low grade lesions, HPV16 DNA clearance was observed in 8 and no recurrence of high grade lesions was observed for 12 months after treatment [61]. Although these data were considered very encouraging, no immunological analysis was reported in this trial and a correlation between vaccine-induced $\mathrm{T}$ cell and viral clearance has not been reported. A subsequent randomized placebo-controlled phase Ilb trial (NCT01022346) in 206 women with $\mathrm{CIN} 2 / 3$ showed greater histological regression in vaccinees than placebos $(25 \%$ vs. $10 \%, p=0.0126)$ as was viral 
clearance (37\% vs. $14 \%)$ six months after vaccination. As vaccine efficacy was well below that of excisional/ ablative therapy Transgene discontinued development of TG4001 in 2012.

\section{(B) Bacterial vectors}

Live attenuated bacterial vectors have the capacity to induce both mucosal and systemic humoral and cellmediated immunity. Upon administration recombinant bacteria are likely to be phagocytosed by macrophages and other APCs [62]. Conserved molecular patterns such as lipopolysaccharides in Gram-negative bacteria, lipoteichoic acid in gram-positive bacteria, peptidoglycans and flagellin are recognized by PRRs and can thus trigger innate immune responses $[63,64]$. Attenuation of bacteria is achieved by deleting essential genes involved in virulence regulatory systems or the aromatic amino acid biosynthesis pathway. These mutations render the bacteria unable to reproduce in the host whilst preserving their capacity to synthesize encoded antigens.

The most commonly used bacterial vector for vaccine development is Listeria monocytogenes (Lm), a Grampositive intracellular facultative anaerobe. Once inside the phagosome $\mathrm{Lm}$ secretes the membrane-active virulence factors LLO and phospholipase C, which degrade the phagolysosomal membrane, enabling entry into the cytoplasm where expressed proteins gain access to the endogenous antigen-processing pathway $[65,66]$. $\mathrm{Lm}$ elicits durable $\mathrm{CD}^{+} \mathrm{T}$ cell responses. Once expressed in a phagosome, Lm-encoded proteins can also be presented directly to $\mathrm{MHC}$ class II molecules [67] and therefore induces both $\mathrm{CD}^{+}$and $\mathrm{CD} 8^{+} \mathrm{T}$ cell responses.

Bacterial vectors are relatively simple and inexpensive to produce, suited to large scale manufacture and stable without refrigeration (via lyophilisation). They have a large capacity for foreign DNA, can be eradicated with antibiotics if an adverse reaction occurs during large scale trials and can be administered orally.

The first clinical trial of a live attenuated bacterial vectored therapeutic HPV vaccine evaluated Lm expressing a HPV16 E7 antigen fused to a fragment of LLO, Lm-LLO-E7/ ADXS11-001, in 15 patients with advanced carcinoma of the cervix who had failed prior chemotherapy, radiotherapy and or surgery. The vaccine was shown to be safe and a reduction in total tumour size was observed in four patients [68]. Three patients showed an increase in E7-specific T cell responses post vaccination. Phase I-II trials are underway in anorectal, head and neck and cervical cancer (NCT02399813, NCT02002182, NCT02291055, NCT01266460). Patients with persistent or 
recurrent metastatic cervical cancer enrolled in a Phase II trial, received ADXS11-001 intravenously, overall 1year survival was $38 \%$, which compares favourably with the expected 12 -month survival of $24 \%$, established using a database of 500 patients with persistent or recurrent metastatic carcinoma of the cervix. A phase III trial (AIM2CERV) is investigating ADXS11-001 administered as adjuvant immunotherapy in patients with high-risk, locally advanced cervical cancer following chemoradiation (NCT02853604).

\section{(B) Peptide and protein based vaccines}

\section{(C) Peptide vaccines}

Peptide-based therapeutic vaccines have the advantages of stability, safety and feasibility of large-scale production [69]. They can be divided into specific epitope (short) peptides and synthetic long peptides (SLPs). Short peptides (<15 amino acids) do not require processing by professional APCs and can therefore bind exogenously to the MHC class I molecules of all nucleated cells [70]. However, presentation without optimal costimulation can result in a tolerising signal and short peptides have been shown to elicit immunological tolerance rather than protective immunity $[71,72]$. SLPs (>20 amino acids) are too long to stabilize MHC molecules so they must be processed and presented by professional APCs $[73,74]$. SLPs typically harbor both $\mathrm{CD}^{+}$and $\mathrm{CD}^{+} \mathrm{T}$ cell epitopes, however, peptide vaccines are poorly immunogenic and therefore require adjuvantation.

PepCan consists of four synthetic peptides covering the HPV16 E6 protein with the Candida skin test reagent as a novel adjuvant. In a single-arm, dose-escalation Phase I clinical trial, 24 women with biopsy-proven CIN2/3 were given four injections intradermally every 3 weeks. At a dose of $50 \mu \mathrm{g}$ a regression rate of $83 \%$ was observed; the overall rate was $52 \%$. Vaccine-induced immune responses to $\mathrm{E} 6$ were detected in $65 \%$ of recipients. A Phase II randomized and double-blinded trial of PepCan for treating cervical high-grade squamous intraepithelial lesions is ongoing (NCT02481414).

Another synthetic long peptide vaccine, ISA101, consists of 13 SLPs spanning the entire HPV16 E6 and E7 oncoproteins. Two Phase I/II trials in women with HPV16 positive high grade VIN, have shown a clinical responses in $>50 \%$ of patients at 12 -months follow-up with $47 \%$ and $30 \%$ of patients having a complete response, respectively $[75,76]$. All responders were still free of disease at 24 months. Spontaneous clearance of HPV16- 
induced high-grade lesions of the vulva is less than 1.5\% [77]. Vaccine-induced T cell responses were present in all patients, and post hoc analyses suggested the magnitude of the HPV16-specific immune response correlated with clinical efficacy of vaccination [76].

\section{(C) Protein based vaccines}

Protein based vaccines include numerous $\mathrm{CD} 4^{+}$and $\mathrm{CD}^{+} \mathrm{T}$ epitopes and avoid the limitation of $\mathrm{MHC}$ restriction which is a disadvantage of short peptide vaccination. However, antigens are preferentially presented via MHC class II, which results in induction of antibodies rather than CTLS. Adjuvantation to enhance endogenous processing, or to target the antigen to DCs is therefore required.

GTL001 (Procervix) comprises HPV16 and HPV18 E7, each fused to detoxified adenylate cyclase from Bordetella pertussis (CyaA) and adjuvanted by imiquimod. The N-terminus of CyaA binds to the adhesion molecule CD11b which is expressed on APCs [78]. A randomised double-blind, placebo-controlled Phase II of GTL001 enrolled 233 patients positive for HPV-16/-18 with either normal or abnormal cervical cytology. All patients received at least one dose of vaccine or placebo along with topical imiquimod and were assessed for viral clearance at 2 years. The difference in viral clearance rates did not reach statistical significance and rates of progression to high-grade lesions were identical in the GTL001 and placebo groups [79] (NCT01957878). Further clinical development was therefore halted.

A second generation vaccine has since been developed. GTL002 comprises modified E7 proteins from HPV16, $18,45,31,33$, and 52. Pre-clinical immunogenicity of GTL002 has been evaluated in inbred and outbred mice and in Beagle dogs. E7-specific T cell responses were induced against each of the six HPV genotypes and in vivo efficacy has been demonstrated using the widely used TC-1 tumour challenge model [80].

\section{(A) How to improve on current approaches}

All the therapeutic vaccines tested in clinical trials to date have been safe and well tolerated. However, they have largely yielded disappointing clinical responses. Human HPV-induced cancers have been largely refractory to approaches shown to be successful in rodents, highlighting the limitations of currently used pre-clinical models. The efficacy of vaccine-induced $T$ cells may be compromised by the numerous mechanisms of 
immunoevasion and immunosuppression exploited by tumours, therefore, treatment modalities that can downmodulate the regulatory mechanisms in the tumour microenvironment need consideration.

\section{(B) Breaking local immune regulation}

Regulatory T cells (Tregs), tumour-associated macrophages, fibroblasts, adipocytes and myeloid-derived suppressor cells present within the tumour microenvironment can inhibit tumour-specific CTLs and NK cells. Tregs suppress T cell expansion and function [81]. The presence of large numbers of Tregs in relation to tumourspecific effector T cells has been associated with poor clinical prognosis in many tumours [82]. The ratio of $\mathrm{CD8}^{+} \mathrm{T}$ :Tregs is an independent prognostic factor in cervical cancer [83]. To improve the success of therapeutic HPV vaccines selective Treg depletion may be needed. Depletion of Tregs by an anti-CD25 antibody before DNAE7 vaccination in tumour-bearing mice enhanced the number of vaccine-induced $C D 8^{+} T$ cells and resulted in enhanced survival [84].

\section{(B) Checkpoint blockade}

Immune checkpoint mechanisms such as cytotoxic T lymphocyte antigen 4 (CTLA-4) and programmed cell death protein 1 (PD-1) prevent excessive and uncontrolled immune responses. CTLA-4 competes with CD28 for binding to the CD80/86 ligands, thereby inhibiting T cell proliferation, blocking cytokine production and promoting $\mathrm{T}$ cell anergy. PD-1 is transiently induced following immune activation. Chronic antigen exposure, such as in tumours, leads to persistently high levels of PD-1. Binding of PD-1 to its ligand, PD-L1, down-regulates T-cell receptor signaling, resulting in T-cell anergy and apoptosis and leading to immune suppression [85]. Many tumours express PD-L1, which may serve as an important mechanism for eradicating tumour reactive T cells within the tumour microenvironment [86]. Similar mechanisms probably also limit the clonal expansion of T cells following vaccination, providing a rationale for combining therapeutic HPV vaccines with checkpoint inhibitors. A phase I/II trial is currently investigating ADXS11-001 and the fully humanized anti-PD-L1 antibody, MEDI4736, alone or in combination in previously treated locally advanced or metastatic cervical or HPV+ head \& neck cancer (NCT02291055). 


\section{(B) Chemo-immunotherapy}

Certain chemotherapeutics can enhance the anti-tumour effect of therapeutic vaccination. Although the precise mechanisms of this synergistic effect have not yet been completely defined, chemotherapy is thought to convert the tumour microenvironment into a site permissive for vaccination by enhancing antigen density and T cell infiltration, and by sensitising tumour cells to cytotoxic $\mathrm{CD}^{+} \mathrm{T}$ cell-mediated killing

A Phase I/II study is underway to assess the safety and tolerability of the SLP vaccine, ISA101, and the immune modulating effects of ISA101 when combined with carboplatin and paclitaxel, with or without bevacizumab. The study aims to recruit 100 women with HPV16 positive advanced or recurrent cervical cancer who have no curative treatment options. Study completion is expected in 2021 (NCT02128126).

\section{(B) Personalised antigens}

An important consideration is whether cancer vaccines that use shared antigens not specific for individual tumours can succeed. The full repertoire of tumour antigens at the individual patient level can now be identified through immune profiling thanks to advances in sequencing technology. Neo-antigens are newly formed mutated proteins that arise as a result of genomic instability of tumours. Both $\mathrm{CD}^{+}$and $\mathrm{CD} 8^{+}$neo-antigenspecific T cells have been identified in multiple human cancers and shown to be associated with a favorable clinical outcome [87]. These neo-antigens could be used in personalised immunotherapy. On obtaining a tumour biopsy, mutations expressed in tumour cells would be identified by whole-exome sequencing. Software algorithms then predict the binding affinity of identified neo-antigens to HLA in order to prioritise the most attractive targets. Prioritised neo-antigens could then be delivered by either adoptive cell transfer or the vaccine platforms discussed earlier. Both approaches could be combined with other immune modulators to overcome immunosuppressive mechanisms in the tumour microenvironment that inhibit neoantigen-specific immune responses. Such an approach showed encouraging results in a patient with a cholangiocarcinoma [88].

\section{(A) Summary}

While progress in the development of targeted therapeutics for HPV disease has been slow, encouraging results from a recent phase IIb trial showing vaccine-induced regression of high-grade CIN lesions are grounds for 
optimism. Further trials to improve on the clinical efficacy of DNA vaccines through adjuvantation are underway. Other promising strategies in development include viral and bacterial vectored vaccines, which offer the advantages of superior potency without the need for separate adjuvants, coupled with excellent safety. However, progress is still hampered by the lack of relevant animal models. Non-human primate species recapitulate some features of human disease but have not been studied in depth.

The historically poor efficacy of therapeutic vaccines in clinical trials could also be due to the immune suppressive nature of the tumour microenvironment which contributes to HPV pathogenesis. Further exploration of the intricate relationship between tumour cells, the tumour microenvironment and immune effectors may reveal pathways that are amenable to manipulation with immune checkpoint inhibitors and other immunomodulators. As HPV-driven cancers express both viral and neo-antigens, the potential for synergy between these agents and therapeutic vaccines should be investigated. 


\section{PRACTICE POINTS}

- HPV-16 and -18 have been identified as the two most prevalent high-risk HPV (hrHPV) types and are accountable for approximately $62 \%$ and $16 \%$, respectively, of invasive cervical cancers.

- Current prophylactic HPV vaccines protect against infection with high risk types HPV16 and 18 and provide limited cross-protection against infection with other hrHPVs. A nonavalent vaccine that provides coverage of an additional five high risk types has been licensed in Europe and the US, but its use is currently limited by cost.

- Prophylactic vaccines are ineffective at clearing pre-existing HPV infections and HPV-associated lesions.

\section{RESEARCH AGENDA}

- Several new platform technologies for antigen delivery have yielded encouraging results in other infectious diseases and are now being applied to the development of therapeutics for HPV disease.

- The principal targets for therapeutics have been E6 and E7; the potential benefits of targeting other viral proteins require further investigation.

- Therapeutic vaccines showing promise in animal models have yielded poor clinical results. The development of more relevant animal models is a priority.

- Tackling the global burden of HPV disease and reaching the most vulnerable populations will require scalable and affordable vaccine manufacturing processes and sustainable delivery in low resourced settings.

\section{ACKNOWLEDGEMENTS}

GH and LD have received financial support from Oxford NIHR Biomedical Research Centre, UK. LD is a Jenner Investigator. 
CONFLICTS OF INTEREST

The authors have no conflicts of interest. 
1. zur Hausen H. Human papillomaviruses and their possible role in squamous cell carcinomas. Curr Top Microbiol Immunol. 1977;78:1-30.

2. Guan P, Howell-Jones R, Li N, Bruni L, de Sanjosé S, Franceschi S, et al. Human papillomavirus types in 115,789 HPV-positive women: a meta-analysis from cervical infection to cancer. Int J cancer. 2012;131:2349-59.

*3. Chaturvedi AK. Beyond cervical cancer: burden of other HPV-related cancers among men and women. J Adolesc Health. 2010;46:S20-6.

4. Herrero R, González P, Markowitz LE. Present status of human papillomavirus vaccine development and implementation. Lancet Oncol. 2015;16:e206-16.

5. Muñoz N, Kjaer SK, Sigurdsson K, Iversen O-E, Hernandez-Avila M, Wheeler CM, et al. Impact of Human Papillomavirus (HPV)-6/11/16/18 Vaccine on All HPV-Associated Genital Diseases in Young Women. JNCI J Natl Cancer Inst. 2010;102:325-39.

*6. Lehtinen M, Paavonen J, Wheeler CM, Jaisamrarn U, Garland SM, Castellsagué X, et al. Overall efficacy of HPV-16/18 AS04-adjuvanted vaccine against grade 3 or greater cervical intraepithelial neoplasia: 4-year end-ofstudy analysis of the randomised, double-blind PATRICIA trial. Lancet Oncol. 2012;13:89-99.

7. WHO | Strategic Advisory Group of Experts (SAGE) on Immunization. WHO. World Health Organization; 2017;

8. WHO | Weekly Epidemiological Record, 2 December 2016, vol. 91, 48 (pp. 561-584). WHO. World Health Organization; 2016;

9. Clifford GM, Smith JS, Plummer M, Muñoz N, Franceschi S. Human papillomavirus types in invasive cervical cancer worldwide: a meta-analysis. Br J Cancer. Nature Publishing Group; 2003;88:63-73.

*10. Brown DR, Kjaer SK, Sigurdsson K, Iversen O, Hernandez-Avila M, Wheeler CM, et al. The Impact of Quadrivalent Human Papillomavirus (HPV; Types 6, 11, 16, and 18) L1 Virus-Like Particle Vaccine on Infection and Disease Due to Oncogenic Nonvaccine HPV Types in Generally HPV-Naive Women Aged 16-26 Years. J Infect Dis. 2009;199:926-35.

*11. Wheeler CM, Kjaer SK, Sigurdsson K, Iversen O, Hernandez-Avila M, Perez G, et al. The Impact of Quadrivalent Human Papillomavirus (HPV; Types 6, 11, 16, and 18) L1 Virus-Like Particle Vaccine on Infection 
and Disease Due to Oncogenic Nonvaccine HPV Types in Sexually Active Women Aged 16-26 Years. J Infect Dis. 2009;199:936-44.

12. Hildesheim A, Herrero R, Wacholder S, Rodriguez AC, Solomon D, Bratti MC, et al. Effect of human papillomavirus 16/18 L1 viruslike particle vaccine among young women with preexisting infection: a randomized trial. JAMA. 2007;298:743-53.

13. Schiller JT, Castellsagué X, Garland SM. A Review of Clinical Trials of Human Papillomavirus Prophylactic Vaccines. Vaccine. 2012;30:F123-38.

14. GLOBOCAN 2012: Estimated Cancer Incidence, Mortality and Prevalence Worldwide in 2012 v1.0

15. Castle PE, Gage JC, Wheeler CM, Schiffman M. The clinical meaning of a cervical intraepithelial neoplasia grade 1 biopsy. Obstet Gynecol. 2011;118:1222-9.

16. Elit L, Levine MN, Julian JA, Sellors JW, Lytwyn A, Chong S, et al. Expectant management versus immediate treatment for low-grade cervical intraepithelial neoplasia : a randomized trial in Canada and Brazil. Cancer. 2011;117:1438-45.

17. Health England P. NHS Cervical Screening Programme Colposcopy and Programme Management Public Health England leads the NHS Screening Programmes. Public Heal Engl. 2016;3:5-108.

18. Martin-Hirsch PP, Paraskevaidis E, Kitchener HC. Surgery for cervical intraepithelial neoplasia. In: MartinHirsch PP, editor. Cochrane Database Syst Rev. Chichester, UK: John Wiley \& Sons, Ltd; 1999

19. Kyrgiou M, Athanasiou A, Paraskevaidi M, Mitra A, Kalliala I, Martin-Hirsch P, et al. Adverse obstetric outcomes after local treatment for cervical preinvasive and early invasive disease according to cone depth: systematic review and meta-analysis. BMJ. 2016;i3633.

20. Kyrgiou M, Mitra A, Arbyn M, Stasinou SM, Martin-Hirsch $P$, Bennett $P$, et al. Fertility and early pregnancy outcomes after treatment for cervical intraepithelial neoplasia: systematic review and meta-analysis. BMJ. 2014;349:g6192-g6192.

21. Phadnis S V, Atilade A, Young MPA, Evans H, Walker PG. The volume perspective: a comparison of two excisional treatments for cervical intraepithelial neoplasia (laser versus LLETZ). BJOG. 2010;117:615-9. 
22. Ginindza TG, Sartorius B, Dlamini X, Östensson E. Cost analysis of Human Papillomavirus-related cervical diseases and genital warts in Swaziland. PLoS One. Public Library of Science; 2017;12.

23. Trimble CL, Piantadosi S, Gravitt P, Ronnett B, Pizer E, Elko A, et al. Spontaneous regression of high-grade cervical dysplasia: effects of human papillomavirus type and HLA phenotype. Clin Cancer Res. 2005;11:4717-23.

24. De Vuyst H, Lillo F, Broutet N, Smith JS. HIV, human papillomavirus, and cervical neoplasia and cancer in the era of highly active antiretroviral therapy. Eur J Cancer Prev. 2008;17:545-54.

25. Hinten F, Meeuwis KAP, van Rossum MM, de Hullu JA. HPV-related (pre)malignancies of the female anogenital tract in renal transplant recipients. Crit Rev Oncol Hematol. 2012;84:161-80.

26. Trimble CL, Clark RA, Thoburn C, Hanson NC, Tassello J, Frosina D, et al. Human papillomavirus 16-associated cervical intraepithelial neoplasia in humans excludes CD8 T cells from dysplastic epithelium. J Immunol. 2010;185:7107-14.

27. Dillon S, Sasagawa T, Crawford A, Prestidge J, Inder MK, Jerram J, et al. Resolution of cervical dysplasia is associated with T-cell proliferative responses to human papillomavirus type 16 E2. J Gen Virol. 2007;88:803-13.

28. Woo YL, van den Hende M, Sterling JC, Coleman N, Crawford RAF, Kwappenberg KMC, et al. A prospective study on the natural course of low-grade squamous intraepithelial lesions and the presence of HPV16 E2-, E6and E7-specific T-cell responses. Int J cancer. 2010;126:133-41.

29. Kim KH, Greenfield WW, Cannon MJ, Coleman HN, Spencer HJ, Nakagawa M. CD4+ T-cell response against human papillomavirus type 16 E6 protein is associated with a favorable clinical trend. Cancer Immunol Immunother. 2012;61:63-70.

30. Steele JC, Mann CH, Rookes S, Rollason T, Murphy D, Freeth MG, et al. T-cell responses to human papillomavirus type 16 among women with different grades of cervical neoplasia. Br J Cancer. 2005;93:248-59.

31. Monnier-Benoit S, Mauny F, Riethmuller D, Guerrini J-S, Căpîlna M, Félix S, et al. Immunohistochemical analysis of $\mathrm{CD} 4+$ and $\mathrm{CD} 8+\mathrm{T}$-cell subsets in high risk human papillomavirus-associated pre-malignant and malignant lesions of the uterine cervix. Gynecol Oncol. 2006;102:22-31.

32. Cao Y, Zhao J, Lei Z, Shen S, Liu C, Li D, et al. Local accumulation of FOXP3+ regulatory T cells: evidence for an 
immune evasion mechanism in patients with large condylomata acuminata. J Immunol. 2008;180:7681-6.

33. Molling JW, de Gruijl TD, Glim J, Moreno M, Rozendaal L, Meijer CJLM, et al. CD4(+)CD25hi regulatory T-cell frequency correlates with persistence of human papillomavirus type 16 and T helper cell responses in patients with cervical intraepithelial neoplasia. Int J cancer. 2007;121:1749-55.

34. Strickler HD, Martinson J, Desai S, Xie X, Burk RD, Anastos K, et al. The Relation of Plasmacytoid Dendritic Cells (pDCs) and Regulatory T-Cells (Tregs) with HPV Persistence in HIV-Infected and HIV-Uninfected Women. Viral Immunol. 2014;27:20-5.

35. Kojima S, Kawana K, Tomio K, Yamashita A, Taguchi A, Miura S, et al. The prevalence of cervical regulatory T cells in HPV-related cervical intraepithelial neoplasia (CIN) correlates inversely with spontaneous regression of CIN. Am J Reprod Immunol. 2013;69:134-41.

36. Stanley MA. Imiquimod and the imidazoquinolones: mechanism of action and therapeutic potential. Clin Exp Dermatol. 2002;27:571-7.

37. Nicholls PK, Moore PF, Anderson DM, Moore RA, Parry NR, Gough GW, et al. Regression of Canine Oral Papillomas Is Associated with Infiltration of CD4+ and CD8+ Lymphocytes. Virology. 2001;283:31-9.

38. Barber GN. Cytoplasmic DNA innate immune pathways. Immunol Rev. 2011;243:99-108.

39. Sheets RL, Stein J, Manetz TS, Duffy C, Nason M, Andrews C, et al. Biodistribution of DNA plasmid vaccines against HIV-1, Ebola, Severe Acute Respiratory Syndrome, or West Nile virus is similar, without integration, despite differing plasmid backbones or gene inserts. Toxicol Sci. 2006;91:610-9.

40. Ledwith BJ, Manam S, Troilo PJ, Barnum AB, Pauley CJ, Griffiths TG, et al. Plasmid DNA vaccines: investigation of integration into host cellular DNA following intramuscular injection in mice. Intervirology. 2000;43:258-72.

41. Dupuis M, Denis-Mize K, Woo C, Goldbeck C, Selby MJ, Chen M, et al. Distribution of DNA vaccines determines their immunogenicity after intramuscular injection in mice. J Immunol. 2000;165:2850-8.

42. Stratford R, Douce G, Zhang-Barber L, Fairweather N, Eskola J, Dougan G. Influence of codon usage on the immunogenicity of a DNA vaccine against tetanus. Vaccine. 2000;19:810-5.

43. Lin C-T, Tsai Y-C, He L, Yeh C-N, Chang T-C, Soong Y-K, et al. DNA vaccines encoding IL-2 linked to HPV-16 E7 
antigen generate enhanced E7-specific CTL responses and antitumor activity. Immunol Lett. NIH Public Access; 2007;114:86-93.

44. Liu J, Kjeken R, Mathiesen I, Barouch DH. Recruitment of Antigen-Presenting Cells to the Site of Inoculation and Augmentation of Human Immunodeficiency Virus Type 1 DNA Vaccine Immunogenicity by In Vivo Electroporation. J Virol. 2008;82:5643-9.

45. de Mare A, Lambeck AJA, Regts J, van Dam GM, Nijman HW, Snippe H, et al. Viral vector-based prime-boost immunization regimens: a possible involvement of T-cell competition. Gene Ther. 2008;15:393-403.

46. Lu S. Heterologous prime-boost vaccination. Curr Opin Immunol. 2009;21:346-51.

*47. Trimble CL, Morrow MP, Kraynyak KA, Shen X, Dallas M, Yan J, et al. Safety, efficacy, and immunogenicity of VGX-3100, a therapeutic synthetic DNA vaccine targeting human papillomavirus 16 and 18 E6 and E7 proteins for cervical intraepithelial neoplasia 2/3: a randomised, double-blind, placebo-controlled phase $2 \mathrm{~b}$ trial. Lancet. 2015;386:2078-88.

48. Diebold SS, Kaisho T, Hemmi H, Akira S, Reis e Sousa C. Innate antiviral responses by means of TLR7-mediated recognition of single-stranded RNA. Science. 2004;303:1529-31.

49. Phua KKL, Leong KW, Nair SK. Transfection efficiency and transgene expression kinetics of mRNA delivered in naked and nanoparticle format. J Control Release. 2013;166:227-33.

50. Ewer KJ, Lambe T, Rollier CS, Spencer AJ, Hill AV, Dorrell L. Viral vectors as vaccine platforms: from immunogenicity to impact. Curr Opin Immunol. 2016;41:47-54.

51. Khan S, Oosterhuis K, Wunderlich K, Bunnik EM, Bhaggoe M, Boedhoe S, et al. Development of a replicationdeficient adenoviral vector-based vaccine candidate for the interception of HPV16- and HPV18-induced infections and disease. Int J Cancer. 2017;141:393-404.

52. Ragonnaud E, Andersson A-MC, Mariya S, Pedersen AG, Burk RD, Folgori A, et al. Therapeutic Vaccine Against Primate Papillomavirus Infections of the Cervix. J Immunother. 40:51-61.

53. Holst PJ, Sorensen MR, Mandrup Jensen CM, Orskov C, Thomsen AR, Christensen JP. MHC class II-associated invariant chain linkage of antigen dramatically improves cell-mediated immunity induced by adenovirus 
vaccines. J Immunol. 2008;180:3339-46.

54. Ragonnaud E, Pedersen AG, Holst PJ. Breadth of T Cell Responses After Immunization with Adenovirus Vectors Encoding Ancestral Antigens or Polyvalent Papillomavirus Antigens. Scand J Immunol. 2017;85:182-90.

55. Moss B. Genetically engineered poxviruses for recombinant gene expression, vaccination, and safety. Proc Natl Acad Sci U S A. 1996;93:11341-8.

56. Moss B. Poxvirus entry and membrane fusion. Virology. 2006;344:48-54.

57. Corona Gutierrez CM, Tinoco A, Navarro T, Contreras ML, Cortes RR, Calzado P, et al. Therapeutic vaccination with MVA E2 can eliminate precancerous lesions (CIN 1, CIN 2, and CIN 3) associated with infection by oncogenic human papillomavirus. Hum Gene Ther. 2004;15:421-31.

58. García-Hernández E, González-Sánchez JL, Andrade-Manzano A, Contreras ML, Padilla S, Guzmán CC, et al. Regression of papilloma high-grade lesions (CIN 2 and CIN 3) is stimulated by therapeutic vaccination with MVA E2 recombinant vaccine. Cancer Gene Ther. 2006;13:592-7.

59. Albarran Y Carvajal A, de la Garza A, Cruz Quiroz BJC, Vazquez Zea E, Díaz Estrada I, Mendez Fuentez E, et al. MVA E2 recombinant vaccine in the treatment of human papillomavirus infection in men presenting intraurethral flat condyloma: a phase I/II study. BioDrugs. 2007;21:47-59.

60. Rosales R, López-Contreras M, Rosales C, Magallanes-Molina J-R, Gonzalez-Vergara R, Arroyo-Cazarez JM, et al. Regression of human papillomavirus intraepithelial lesions is induced by MVA E2 therapeutic vaccine. Hum Gene Ther. Mary Ann Liebert, Inc.; 2014;25:1035-49.

61. Brun J-L, Dalstein V, Leveque J, Mathevet P, Raulic P, Baldauf J-J, et al. Regression of high-grade cervical intraepithelial neoplasia with TG4001 targeted immunotherapy. Am J Obstet Gynecol. 2011;204:169.e1-8.

62. Cossart P, Pizarro-Cerdá J, Lecuit M. Invasion of mammalian cells by Listeria monocytogenes: functional mimicry to subvert cellular functions. Trends Cell Biol. 2003;13:23-31.

63. Dietrich G, Spreng S, Favre D, Viret J-F, Guzman CA. Live attenuated bacteria as vectors to deliver plasmid DNA vaccines. Curr Opin Mol Ther. 2003;5:10-9.

64. Janeway CA, Medzhitov R. I NNATE I MMUNE R ECOGNITION. Annu Rev Immunol. 2002;20:197-216. 
65. Schaible UE, Collins HL, Kaufmann SH. Confrontation between intracellular bacteria and the immune system. Adv Immunol. 1999;71:267-377.

66. Mollenkopf H, Dietrich G, Kaufmann SH. Intracellular bacteria as targets and carriers for vaccination. Biol Chem. 2001;382:521-32.

67. Watts $C$. The exogenous pathway for antigen presentation on major histocompatibility complex class II and CD1 molecules. Nat Immunol. 2004;5:685-92.

68. Maciag PC, Radulovic S, Rothman J. The first clinical use of a live-attenuated Listeria monocytogenes vaccine: A Phase I safety study of Lm-LLO-E7 in patients with advanced carcinoma of the cervix. Vaccine. 2009;27:397583.

69. Hung C-F, Wu TC, Monie A, Roden R. Antigen-specific immunotherapy of cervical and ovarian cancer. Immunol Rev. 2008;222:43-69.

70. van der Burg SH, Bijker MS, Welters MJP, Offringa R, Melief CJM. Improved peptide vaccine strategies, creating synthetic artificial infections to maximize immune efficacy. Adv Drug Deliv Rev. 2006;58:916-30.

71. Toes RE, Blom RJ, Offringa R, Kast WM, Melief CJ. Enhanced tumor outgrowth after peptide vaccination. Functional deletion of tumor-specific CTL induced by peptide vaccination can lead to the inability to reject tumors. J Immunol. 1996;156:3911-8.

72. Toes RE, Offringa R, Blom RJ, Melief CJ, Kast WM. Peptide vaccination can lead to enhanced tumor growth through specific T-cell tolerance induction. Proc Natl Acad Sci U S A. 1996;93:7855-60.

73. Sercarz EE, Maverakis E. Mhc-guided processing: binding of large antigen fragments. Nat Rev Immunol. 2003;3:621-9.

74. Bijker MS, van den Eeden SJF, Franken KL, Melief CJM, van der Burg SH, Offringa R. Superior induction of anti-tumor CTL immunity by extended peptide vaccines involves prolonged, DC-focused antigen presentation. Eur J Immunol. 2008;38:1033-42.

75. Kenter GG, Welters MJP, Valentijn ARPM, Lowik MJG, Berends-van der Meer DMA, Vloon APG, et al. Vaccination against HPV-16 Oncoproteins for Vulvar Intraepithelial Neoplasia. N Engl J Med. 2009;361:1838-47. 
76. van Poelgeest MIE, Welters MJP, Vermeij R, Stynenbosch LFM, Loof NM, Berends-van der Meer DMA, et al. Vaccination against Oncoproteins of HPV16 for Noninvasive Vulvar/Vaginal Lesions: Lesion Clearance Is Related to the Strength of the T-Cell Response. Clin Cancer Res. 2016;22:2342-50.

77. van Seters M, van Beurden M, de Craen AJM, Vloon A, Lowik M, Meer DB der, et al. Is the assumed natural history of vulvar intraepithelial neoplasia III based on enough evidence? A systematic review of 3322 published patients. Gynecol Oncol. BioMed Central; 2005;97:645-51.

78. Simsova $M$, Sebo $P$, Leclerc $C$. The adenylate cyclase toxin from Bordetella pertussis--a novel promising vehicle for antigen delivery to dendritic cells. Int J Med Microbiol. 2004;293:571-6.

79. Genticel Reports Final Results of GTL001 Phase 2 Trial in HPV16/18-Infected Women Brussels Stock Exchange:GTCL

80. M. Esquerré 1, Y. Misseri 1, E. Totain 2, G. Zingone 1, N. Cohet 1, P. Bridonneau 1, C. Gonindard 1, M. Best 1, A. Navarro 1, J. Morato 1, H. Keller 1, A. Bousquet-Mélou 2, S. Boullier 2 MCB 1. PRECLINICAL PROOF OF CONCEPT OF GTL002, A MULTIVALENT CANDIDATE FOR THE IMMUNOTHERAPY OF HUMAN PAPILLOMA VIRUS HPV16/18/45/31/33/52-INFECTED WOMEN. EUROGIN. 2016. p. 123.

81. van der Burg SH, Piersma SJ, de Jong A, van der Hulst JM, Kwappenberg KMC, van den Hende M, et al. Association of cervical cancer with the presence of CD4+ regulatory T cells specific for human papillomavirus antigens. Proc Natl Acad Sci U S A. 2007;104:12087-92.

82. Piersma SJ, Welters MJP, van der Burg SH. Tumor-specific regulatory T cells in cancer patients. Hum Immunol. 2008;69:241-9.

83. Jordanova ES, Gorter A, Ayachi O, Prins F, Durrant LG, Kenter GG, et al. Human leukocyte antigen class I, MHC class I chain-related molecule A, and CD8+/regulatory T-cell ratio: which variable determines survival of cervical cancer patients? Clin Cancer Res. 2008;14:2028-35.

84. Chuang C-M, Hoory T, Monie A, Wu A, Wang M-C, Hung C-F. Enhancing therapeutic HPV DNA vaccine potency through depletion of CD4+CD25+ T regulatory cells. Vaccine. 2009;27:684-9.

85. Francisco LM, Sage PT, Sharpe AH. The PD-1 pathway in tolerance and autoimmunity. Immunol Rev. NIH Public Access; 2010;236:219-42. 
86. Dong H, Strome SE, Salomao DR, Tamura H, Hirano F, Flies DB, et al. Tumor-associated B7-H1 promotes Tcell apoptosis: A potential mechanism of immune evasion. Nat Med. 2002;8:793-800.

87. Bobisse S, Foukas PG, Coukos G, Harari A. Neoantigen-based cancer immunotherapy. Ann Transl Med. AME Publications; 2016;4:262.

*88. Tran E, Turcotte S, Gros A, Robbins PF, Lu Y-C, Dudley ME, et al. Cancer Immunotherapy Based on MutationSpecific CD4+ T Cells in a Patient with Epithelial Cancer. Science (80- ). 2014;344:641-5. 